\title{
Articulação teórico-prática na formação do enfermeiro
}

\section{Cássia Regina Fernandes Biffe Peres', Sílvia Franco da Rocha Tonhom $^{2}$, Márcia Aparecida Padovan Otani ${ }^{2}$, Carina Rejane Fernandes Biffe $^{3}$ e Maria José Sanches Marin²}

\author{
${ }^{1}$ Faculdade de Medicina de Marília; Curso de Medicina Fundação Educacional do Município \\ de Assis, Brasil | c.r.biffe@gmail.com | https://orcid.org/0000-0002-8430-0400 \\ ${ }^{2}$ Faculdade de Medicina de Marília, Brasil | siltonhom@gmail.com; \\ mpadovanotani@gmail.com; marnadia@terra.com.br | http://orcid.org/ 0000-0001-7522- \\ 2861; https://orcid.org/0000-0001-9540-4996; https://orcid.org/0000-0001-6210-6941 \\ ${ }^{3}$ Grupo de Vigilância Epidemiológica da Secretaria de Estado da Saúde de São Paulo, \\ Brasil | carina_biffe@yahoo.com.br | https://orcid.org/0000-0001-5090-7987
}

\begin{abstract}
Resumo: Introdução: historicamente, as ações de saúde pautaram-se no cuidado biológico, fragmentado, hospitalar e especializado. A formação do enfermeiro manteve a premissa de que a teoria deve anteceder a prática, com currículos organizados por disciplinas. No entanto, essa organização curricular vem sendo questionada. As Diretrizes Curriculares Nacionais, apontam para a necessidade da formação por competência, integralidade do cuidado, olhar ampliado do processo saúde-doença e da capacidade de atender as necessidades da população. Nesse sentindo, as Instituições de Ensino devem aproximar os estudantes ao mundo do trabalho, buscando superar o desafio de articular teoria e prática. Para essa compreensão, partiu-se da pergunta: como se dá a articulação teórico-prática na graduação em enfermagem? Objetivo: analisar como está ocorrendo a articulação teórico-prática na formação do enfermeiro no estado de São Paulo-Brasil. Método: estudo qualitativo fundamentado na hermenêutica-dialética, que analisou entrevistas de 21 professores de seis cursos de enfermagem. Resultados: observou-se, por um lado, aspectos que comprometem a articulação teórico-prática, como a organização curricular por disciplinas, a falta de definição dos conteúdos básicos necessários à formação do enfermeiro e a dicotomia entre ciclo básico e profissional. Em contrapartida, evidenciou-se um caminhar para efetivar essa articulação constatado pelo reconhecimento da importância do planejamento curricular conjunto e por iniciativas de currículo integrado, com construção do conhecimento a partir do mundo do trabalho. Conclusões: a articulação teórico-prática representa grande desafio na formação do enfermeiro. Seu enfrentamento pressupõe mudanças paradigmáticas nos currículos e, consequentemente, na forma de pensar e agir dos atores envolvidos no processo de ensinoaprendizagem, visando à praxis.
\end{abstract}

Palavras-chave: Educação em Enfermagem; Currículo; Prática Profissional; Competência Profissional.

\section{Theoretical-Practical Articulation in the Training of Nurses}

Abstract: Introduction: historically, health actions were guided by biological, fragmented, hospital and specialized care. The training of nurses maintained the premise that theory must precede practice, with curricula organized by disciplines. However, this curricular organization has been questioned. The National Curriculum Guidelines point to the need for training by competence, comprehensive care, an expanded look at the health-disease process and the ability to meet the needs of the population. In this sense, Teaching Institutions must bring students closer to the world of work, seeking to overcome the challenge of articulating theory with practice. For this understanding, we started with the question: how does the theoreticalpractical articulation take place in undergraduate nursing? Goal: to analyze how the theoretical-practical articulation is taking place in the training of nurses in the state of São Paulo-Brazil; Method: qualitative study based on hermeneutics-dialectics, which analyzed interviews of 21 teachers from six nursing courses. Result: it was observed, on the one hand, aspects that compromise the theoretical-practical articulation, such as the curricular organization by subjects, the lack of definition of the basic contents necessary for the training of nurses and the dichotomy between basic and professional cycle. On the other hand, it was evident that there was a path to achieve this articulation, as evidenced by the recognition of the importance of joint curricular planning and by integrated curriculum initiatives, with the construction of knowledge from the world of work. Conclusions: the theoretical-practical articulation represents a great challenge in the training of nurses. Their confrontation presupposes paradigmatic changes in the curricula and, consequently, in the way of thinking and acting of the actors involved in the teaching-learning process, aiming at praxis.

Keywords: Education; Nursing; Curriculum; Professional Practice; Professional Competence. 


\section{Introdução}

Historicamente as ações de saúde pautaram-se no cuidado biológico, fragmentado, hospitalar e especializado, inspirado no modelo flexneriano, repercutindo na formação dos profissionais de saúde (Ximenes Neto et al., 2020). Nessa perspectiva, a educação em enfermagem manteve a premissa de que a teoria deve anteceder a prática, com currículos organizados em disciplinas.

Essa organização vem sendo questionada, especialmente pelo aumento de produção de conhecimento e tecnologias, o que demanda incorporação de novos saberes ao fazer profissional.

As Diretrizes Curriculares para os Cursos de Enfermagem (DCN) direcionam para formação por competência, com perfil profissional voltado à integralidade do cuidado, envolvendo visão ampliada do processo saúde-doença, na lógica interdisciplinar e compreensão do sistema de saúde em rede (Ministério da Educação, 2001).

Para esse complexo direcionamento, as organizações curriculares devem aproximar a escola ao mundo do trabalho, proporcionar a prática reflexiva para a transformação da realidade, reforçando a articulação da teoria com a prática e a mobilização dos atributos afetivos, cognitivos e psicomotores (Tonhom et al., 2014).

Neste sentido, resgata-se o conceito de práxis, teorizada por Marx e aprofundada na educação por Paulo Freire (2013), como a capacidade de o sujeito atuar e refletir, possibilitando a transformação da realidade conforme as finalidades delineadas, ou seja, a possibilidade de agir, refletir sobre sua ação e retornar à realidade.

Nessa direção, os cursos de graduação em Enfermagem têm organizado suas matrizes curriculares com diferentes aproximações da prática. Encontram-se currículos por disciplinas; em ciclos, fundamental, composicional e profissional, com inserção progressiva na prática; atividades teóricas e práticas desde as séries iniciais com outros cursos da saúde e organização curricular por competência, com métodos ativos de ensinoaprendizagem (Soriano et al., 2015).

Apesar dos avanços proporcionados pelas DCN, a articulação teórico-prática na formação em Enfermagem ainda é um desafio por dificuldades históricas e contemporâneas. (Ximenes Neto et al., 2020).

Para ampliar essa compreensão, partiu-se da questão: Como se dá a articulação teóricoprática no ensino de graduação em Enfermagem?

Assim, tem-se como objetivo analisar como está ocorrendo a articulação teórico-prática na formação do enfermeiro no estado de São Paulo-Brasil.

\section{Metodologia}

Pesquisa qualitativa, fundamentada nos pressupostos da hermenêutica-dialética. Abordagem que propõe contextualizar o discurso dos atores sobre as questões socioeconômicas, políticas e sociais, em busca de sua compreensão e autenticidade (Minayo et al., 2016).

O referencial teórico-metodológico, pautado na hermenêutica-dialética, ancorou-se nos princípios e diretrizes do Sistema Único de Saúde e nas DCN, o que possibilitou a compreensão dos fenômenos na sua complexidade e historicidade.

A perspectiva hermenêutica preocupa-se com a compreensão do texto, que pode ser oriundo de biografia, narrativa, entrevistas, documentos, livro, entre outros. Tendo como unidade temporal o presente, "marca o encontro entre o presente e o passado, ou entre o diferente e a diversidade dentro da vida atual, mediada pela linguagem". Reconhece-se, no entanto, que a linguagem nem sempre é totalmente transparente em si mesma, com possibilidade de entendimento parcial ou de não entendimento (Minayo, 2014). 
No entanto, enxergam-se limites na fundamentação de uma interpretação emancipatória dos fatos humanos, uma vez que não incorpora movimento da crítica na reconstrução do discurso e do seu sentido prático. Assim, aplica-se à hermenêutica uma visão dialética, o que constitui a hermenêutica-dialética (Minayo, 2014).

A dialética incorpora a ideia de crítica, de negação, de oposição, de mudança, de processo, de contradição, de movimento e de transformação da realidade social, favorecendo múltiplas possibilidades de interpretação e compreensão do texto analisado (Minayo, 2014).

A união da hermenêutica e da dialética para a compreensão e crítica da realidade se complementam no momento em que a primeira permite o entendimento dos textos, fatos históricos, cotidianidade e realidade na qual ocorrem e a segunda, por sublinhar a diferença, o contraste, o dissenso e a ruptura do sentido, sendo que, em conjunto, representam um movimento necessário à produção de racionalidade em relação aos processos sociais constituídos de complexidade (Minayo, 2014; Brito et al., 2007).

O estudo foi realizado em seis Instituições de Ensino Superior (IES) que oferecem curso de Enfermagem no estado de São Paulo-Brasil, sendo três públicas e três privadas. Para definição da amostra dos cursos de instituições privadas incluíram-se aqueles com nota três ou mais no Sistema Nacional de Avaliação da Educação Superior (SINAES), considerando-se que não atingir tal conceito representa fragilidade para essa formação profissional. Dos 178 cursos de Enfermagem de escolas privadas do Estado de São Paulo-Brasil, somente seis $(3,4 \%)$ haviam obtido conceito cinco; $20(11,2 \%)$ - conceito quatro e $46(25,8 \%)$ - conceito três, totalizando-se 72 cursos de instituições privadas (Ministério da Educação, 2015). Em relação às instituições públicas, foi incluída sua totalidade, ou seja, nove, independente do conceito atribuído pelo SINAES, pois muitas delas são reguladas pela Secretaria de Estado, e não têm a obrigatoriedade de avaliação pelo Ministério da Educação.

Posteriormente, procedeu-se a seleção da amostra, considerando como critério ter no mínimo dez anos de funcionamento por ser esse um período razoável para desenvolvimento de processos de mudanças a fim de atender às DCN e às demandas da Política Nacional de Saúde. Assim, obteve-se um total de 25 cursos.

Depois de aplicados os dois critérios de inclusão, foram sorteados três cursos de instituição pública e três de instituição privada, com a intenção de buscar a diversidade organizativa das IES.

Os participantes do estudo foram professores enfermeiros das seis IES, sendo todos convidados a participar via e-maile a escolha ocorreu conforme o recebimento da resposta positiva. A saturação teórica se deu com a análise da 21르 entrevista (Minayo, 2017), momento em que as entrevistas não apresentavam informações novas.

A coleta de dados foi realizada por meio de entrevista, com a seguinte questão aberta Como ocorre a articulação teórico-prática na sua prática docente de formação do enfermeiro? - As entrevistas foram realizadas, pela primeira autora, no período de agosto de 2015 a março de 2016, com duração média de 30 minutos, agendadas previamente e realizadas no melhor momento e local para os participantes, gravadas em áudio e transcritas na íntegra pela referida pesquisadora. Para garantir o anonimato das IES e dos participantes, as entrevistas foram codificadas, IES pública - "P"; IES privada - "PRI" e professor -"PRO", acompanhados de sequência numérica.

Considerando que, em todo processo de mudança, há um movimento dialético, a perspectiva hermenêutica-dialética permitiu evidenciar os aspectos relacionados à articulação teórico-prática na formação do enfermeiro.

O método de análise hermenêutico-dialética é descrito como uma forma de reflexão, um caminho do pensamento, norteado pela aproximação com a práxis social, aprofundado na práxis histórica, econômica e política, na busca de uma visão de totalidade para a compreensão do fenômeno. 
Compreende-se, portanto, que o investigador deve imergir no texto na busca de desvelar o que ficou inconsciente para o autor, bem como criar relevâncias e acrescentar conhecimento novo (Brito et al., 2007). Nessa direção, os dados foram interpretados, por todas as autoras, a partir do caminho proposto por Minayo (2014).

No primeiro momento, ocorreu a "ordenação dos dados", incluindo a organização do material; a transcrição do áudio das entrevistas; a releitura do material e organização dos relatos em determinada ordem, o que permitiu o início da classificação.

Num segundo momento, considerado como "classificação dos dados", houve a compreensão de que os dados não existem por si só, eles são construídos a partir de questionamentos sobre eles, com base nos fundamentos teóricos. Por meio de leitura exaustiva dos relatos, foram identificadas as estruturas relevantes das narrativas dos participantes, as quais foram agrupadas em núcleos de sentido, o que possibilitou o estabelecimento das categorias empíricas, que foram confrontadas com as categorias analíticas estabelecidas para balizar a investigação, buscando as relações dialéticas entre elas.

Por fim, realizou-se a "análise final", momento em que se articulou os dados coletados e os referenciais teóricos da pesquisa, num movimento dialético, que considerou a divergência, a contradição, o concreto e o abstrato, o particular e o geral, visando chegar ao concreto pensado e suas interfaces com a articulação teórico-prática na formação do enfermeiro (Minayo et al., 2016).

O estudo foi aprovado pelo Comitê de Ética em Pesquisa Envolvendo Seres Humanos da Faculdade de Medicina de Marília, com parecer número no 816.301.

\section{Resultados}

A maioria dos entrevistados era do sexo feminino, 19 (90,5\%), com idade entre 32 e 59 anos, sendo que 12 (57\%) tinham mais de 50 anos. O tempo de formado variou de nove a 36 anos e o de trabalho na IES era de dois a 28 anos. Quanto à titulação, cinco $(23,8 \%)$ eram especialistas, sete $(33,3 \%)$ mestres, oito $(38,1 \%)$ doutores e um $(4,8 \%)$ livre docente.

$\mathrm{Na}$ análise das entrevistas encontram-se, por um lado, aspectos que comprometem a articulação entre teoria e prática, por outro, há o reconhecimento da necessidade de mudanças e de movimentos consistentes para essa articulação, demonstando um constante movimento dialético.

\subsection{Desarticulação entre a Teoria e a Prática}

Nessa temática, serão apresentados os aspectos que indicam a desarticulação entre teoria e a prática.

Conforme as falas dos participantes, a manutenção e a apresentação de matrizes curriculares por disciplinas distanciam a teoria da prática, pois dificultam discussões mais ampliadas.

Quando uma matriz ainda é organizada por disciplinas, a gente corre um sério risco de fragmentação do processo de ensino aprendizado (PRO3/PR1).

O próprio modelo de currículo não possibilita uma discussão, um desenvolvimento desse ensino-aprendizagem mais integrado (PRO2/P2).

Outro aspecto se refere à indefinição dos conteúdos básicos necessários à formação do enfermeiro, visto que a seleção dos mesmos é realizada por professores de disciplinas básicas, que, na maioria, são de outras categorias profissionais e têm pouca aproximação com o papel do enfermeiro. Assim, os conteúdos necessários são minimizados, sem abordagem aprofundada dos conteúdos referentes às disciplinas profissionalizantes. 
Há a necessidade de discutir qual a real demanda da escola de Enfermagem para aquela disciplina específica. Quem está ensinando anatomia para Enfermagem não pode achar que a Enfermagem é menor. A Enfermagem tem algumas necessidades especificas de aprendizado (PRO1/P3).

Apesar da aproximação dos estudantes com a prática em séries iniciais, a vivência do papel profissional ocorre, de fato, no último ano de graduação, ao realizar o Estágio Curricular Supervisionado (ECS).

Ele vai no $1^{\circ}$ Semestre, apenas para visitas, conhecer os serviços de saúde, concentra mesmo os estágios nos últimos dois anos (PRO1/PRI1).

A falta de articulação da teoria com prática no decorrer da formação pode fazer com que, ao inserir o estudante no campo de estágio, haja necessidade de resgatar a teoria, se essa não foi significativa para o aprendizado no momento em que foi trabalhada.

A gente faz esse resgate na primeira semana, antes de começar a prática, tem se mostrado suficiente (PRO3/PRI2).

Mesmo o aluno que foi muito bem nestas disciplinas, quando você entra de novo nessa disciplina, vai mostrar para ele por que precisa ser daquele jeito e não do outro. Opa, vamos ter que voltar (PRO1/PRI2).

Passa meio despercebida muita coisa e eles só vão dar valor no último ano mesmo. Naquele momento não faz sentido (PRO4/PRI3).

Quanto às dificuldades na articulação, apontam essencialmente a falta de espaços formais de discussão e a sobrecarga dos professores das disciplinas básicas por outras atividades.

Não tem essa integração, acaba conversando meio que de corredor o que já foi trabalhado, o que não foi e como foi (PRO1/PRI3).

São sobrecarregados com outras atividades, pessoal das Cadeiras Básicas trabalha muito com pesquisa, não tem um tempo que sobra, isso acaba dificultando um pouco (PRO2/P2).

\subsection{Perspectivas para a Articulação entre a Teoria e a Prática}

Essa temática aborda os aspectos que levam às perspectivas para a articulação entre a teoria e a prática.

Entre os entrevistados, destaca-se o reconhecimento da necessidade de articulação da teoria com a prática e da aproximação entre os professores das disciplinas básicas e profissionalizantes.

É como se tivesse uma separação, um ciclo básico de um lado e esse ciclo profissional do outro. Mas a ideia é que isso aconteça junto e de uma forma integrada, mas ainda não conseguimos (PRO1/PRI1).

Eles [professores das disciplinas básicas] precisam se aproximar da gente para que a gente possa passar minimamente tudo o que a gente precisa (PRO1/P3).

Encontra-se também o relato de que articulação com as disciplinas básicas acontece em momentos pontuais, em que os professores são chamados para contribuir em determinadas aulas.

Tem situações mais pontuais na discussão de caso, numa determinada aula que o pessoal é chamado (PRO2/P2).

Foram observados ainda integração da teoria com a prática que ocorrem a partir de vivências dos estudantes na prática profissional ou a partir de situações elaboradas previamente. 
A partir das situações vivenciadas pelo estudante ou das situações colocadas previamente elaboradas, se pudessem identificar quais os conhecimentos necessários para que ele pudesse se desenvolver naquela situação. E aí eles buscam os conhecimentos que são necessários das várias áreas, seja do ciclo básico ou do clínico (PRO1/P1).

No depoimento a seguir, tem-se o reconhecimento de que o trabalho articulado com as disciplinas básicas enriquece o contexto do problema e estimula o questionamento dos estudantes.

No planejamento das unidades educacionais tem a participação das cadeiras básicas. Isso ajudou muito nesse movimento de articular as coisas. Aí fica muito rico porque o contexto do problema tem que estimular os questionamentos dos estudantes (PRO4/P1).

\section{Discussão}

Conforme relatado pelos participantes, os currículos organizados por disciplinas desfavorecem a articulação teórico-prática, visto que são desenvolvidas isoladamente e a maioria delas em momentos em que não há inserção em cenários de prática. Assim, fazse necessário que os estudantes resgatem em momento oportuno os conteúdos trabalhados inicialmente, corroborando o estudo que aponta a necessidade de esse resgate teórico e sua articulação com a prática ser realizado pelo professor que se encarrega dos conteúdos profissionalizantes (Campos et al., 2009).

Utilizando-se da perspectiva hermenêutica-dialética para compreender e analisar a realidade, levando em conta os aspectos históricos e a situação atual da articulação teórico-prática nos cursos de Enfermagem, entende-se que, de certa forma o distanciamento entre teoria e prática nos currículos dos cursos de Enfermagem, encontra reforço nas DCN a partir do entendimento de que o ECS deve ser realizado no último ano da formação (Ministério da Educação, 2001). Nos demais momentos do curso, o currículo é desenvolvido com aproximações que nem sempre possibilitam o movimento de agir, refletir e retornar à ação (Ximenes Neto et al., 2020). Embora o ECS traga avanço na aproximação do estudante com o mundo do trabalho (Marran, et al., 2015), em alguns currículos, esse é o único momento em que o estudante é inserido na prática, conforme relatado pelos participantes do estudo. Essa situação revela clara dicotomia entre ciclo básico e profissional.

Aliado a isso, a organização curricular por disciplina dificulta a construção de matrizes articuladas, à medida que cada disciplina atua isoladamente, sem espaços para discussões e planejamento conjunto.

Nesse sentido, mesmo que haja esforços para a interlocução entre as disciplinas, a simples justaposição de conteúdos não garante a ruptura da dicotomia entre teoria e prática. Torna-se necessário integrar os diferentes conhecimentos que fundamentam as práticas do enfermeiro (Campos, et al., 2009). Cabe destacar que a definição de conteúdos realmente importantes à sua formação também é citada pelos entrevistados visto que, na história da criação das escolas de Enfermagem, os conteúdos foram organizados a partir dos currículos dos cursos de medicina, sem a devida clareza do que realmente tem significado para a Enfermagem (Kletemberg \& Siqueira, 2003).

Destarte, a construção de PPC com currículo integrado favorece a contextualização dos saberes, a articulação ensino-serviço-comunidade com problematização e a aproximação da práxis (Peres et al., 2018). Ressalta-se que, em currículos com abordagens dialógicas, essa dificuldade é minimizada, a partir do momento em que o aprendizado se dá na prática e as lacunas de conhecimento são estudadas para se dar conta da situação vivenciada (Tonhom et al., 2014). 
Considera-se, que a estratégia de articular ensino e trabalho é um grande desafio dada a inovação tecnológica frente aos currículos tradicionais e que, de acordo com Lima et al.(2018), na área da saúde, a educação deve ser inserida no contexto do SUS. Ademais, as políticas para superação da fragmentação curricular e implantação de métodos ativos de ensino-aprendizagem, fundamentadas nas DCN, esbarra na cristalização das práticas pedagógicas e no desejo de preservação dos papéis assumidos por escolas e professores (Lima et al., 2018; Lara et al., 2019).

Para superar esse desafio torna-se necessária a adoção de modelos curriculares inovadores, com mudanças na organização curricular e, sobretudo, nos papéis desempenhados por estudantes e professores no processo ensino-aprendizagem. O professor deve desafiar-se a se destituir da detenção do saber e assumir a facilitação do processo de ensino-aprendizagem, favorecendo a construção do conhecimento a partir das necessidades do estudante, estimulando a proatividade, apreendendo novos saberes e ressignificando os existentes (Araújo et al. 2018). Além de se colocarem como aprendizes do processo. Salienta-se que a aprendizagem significativa considera a afetividade do estudante, pois para aprender de maneira significativa não basta a potência das situações de aprendizagem, visto que deve haver disponibilidade do estudante em relacionar o novo conteúdo ao seu conhecimento prévio (Camejo \& Diez, 2016).

Acresce-se a isso a necessidade de espaços formais de construção coletiva dos PPC e planejamento das atividades. É relevante investir na articulação interna, como também, em gestão compartilhada na formação em saúde, para além dos muros da universidade, considerando outros setores da sociedade, e, assim, oportunizar maior efetivação da articulação teoria-prática (Mattia et al., 2018).

Em uma proposta de currículo integrado, alguns aspectos devem ser considerados, como a clareza na definição dos desempenhos esperados para cada série e ao final do curso, além da construção do conhecimento a partir da reflexão sobre a prática vivida, considerando o contexto de vida das pessoas (Lima, 2017), com proposta de avaliação focada no processo e não somente no resultado. O planejamento coletivo é essencial para a representatividade do produto, que possa ser operacionalizado e respeitado por todos (Peres et al., 2018; Lima et al., 2018).

Assim, para que a construção curricular possibilite um contexto práxico, conforme discute Freire (2013), além do processo de reconstrução do PPC, com a produção do conhecimento a partir do mundo do trabalho, nos diferentes momentos da formação, professor e estudante devem desconstruir modelos tradicionais de ensino e ressignificar 0 seu papel em constante movimento dialético para impulsionar a ação (Paro, et al. 2020).

\section{Conclusões}

Os pressupostos da hermenêutica-dialética possibilitaram a compreensão do complexo movimento que as IES têm realizado para a articulação teórico-prática na formação do enfermeiro.

Observa-se que a organização curricular por disciplinas e clara dicotomia em ciclo básico e profissional reforça a fragmentação teórico-prática, o que pode relacionar-se à ausência de planejamento curricular conjunto. Há o reconhecimento da importância de aproximação entre professores de disciplinas básicas e profissionalizantes para a qualificação da formação.

Evidencia-se um caminhar em direção à articulação teórico-prática, com matrizes curriculares integradas à construção do conhecimento a partir do mundo do trabalho.

Compreende-se que embora avanços sejam observados, as mudanças curriculares dos cursos de Enfermagem caminham com dificuldades para a efetiva práxis transformadora, necessária ao atual modelo de atenção à saúde. Assim, depreende-se que há que se investir em currículos que favoreçam a aprendizagem significativa, com a construção do conhecimento a partir e para a prática profissional, com participação ativa de estudantes, professores e profissionais do serviço no processo de ensino e de cuidado. 


\section{Referências}

Araújo, B. B. M.I., Machado, A. C. C., Rossi, C. S., Pacheco, S. T. A. B. \& Rodrigues, M. R. D. (2018) Referencial teórico-metodológico de Paulo Freire: contribuições no campo da enfermagem. Revista de Enfermagem da UERJ, 26, e27310. doi:10.12957/reuerj.2018.27310

Brito, R. M., Santos, E. O., Braga, G. B., Printes, J. S., Chaves, R. M. T., Silva, W. L. A. (2007). A hermenêutica e o processo de construção do conhecimento. Dialógica,1(3), 1-12. http://dialogica.ufam.edu.br/PDF/no3/Rosa_Britto_Hermeneutica.pdf

Campos, C. M. S., Soares, A. B., Trapé, C. A., Silva, B. R. B. \& Silva,T. C. (2009). The relationship theory-practice and the teaching-learning process in a Collective Health Nursing Course. Revista da Escola de Enfermagem da USP, 43(esp 2), 1226-1231.

Camejo, I. \& Diez, D. (2016). Meaningful learning: underlying concept of Cognitive Learning Theory Multimedia? Revista Investimento, 40(89), 68-89. http://ve.scielo.org/scielo.php?script=sci_arttext\&pid=S1010-29142016000300004\&lng=es\&tlng=

Freire, P. (2013). Pedagogia do oprimido (55 $5^{\underline{a}}$ ed.). Paz e Terra.

Kletemberg, D. F. \& Siqueira, M. T. A. D. (2003). The creation of the nursing teaching in Brazil. Cogitare Enfermagem, 8(2), 61-67. doi.org/10.5380/ce.v8i2.1695

Lara, E. M. O., Lima, V. V., Mendes, J. D., Ribeiro, E. C. O. \& Padilha, R. Q. (2019). The teacher in active methodologies and the nuances between teaching and learning: challenges and possibilities. Interface, 23, e180393. doi.org/10.1590/interface.180393

Lima, V. V. (2017). Espiral construtivista: uma metodologia ativa de ensino-aprendizagem. Interface, 21(61), 421-434. doi.org/10.1590/1807-57622016.0316

Lima, V. V., Ribeiro, E. C. O., Padilha, R.Q. \& Mourthé Júnior C. A. (2018). Challenges in the education of health professionals: an interdisciplinary and interprofessional approach. Interface, 22(sup 2), 1549-1562. doi.org/10.1590/1807-57622017.0722

Marran, A. L., Lima, P. G. \& Bagnato, M. H. S. (2015). Educational policies and the supervised curricular internship in the undergraduate nursing course. Trabalho Educação e Saúde, 13(1), 89-108. doi.org/10.1590/1981-7746-sip00025

Mattia, B. J., Kleba, M. E. \& Prado, M. L. (2018). Nursing training and professional practice: an integrative review of literature. Revista Brasileira de Enfermagem, 71(4), 2039-2049. doi.org/10.1590/00347167-2016-0504

Minayo, M. C. S. (2014). O desafio do conhecimento: pesquisa qualitativa em saúde (14를. ed.). Hucitec.

Minayo, M. C. S. (Org), Deslandes, S. F. \& Gomes, R. (2016). Pesquisa social: teoria, método e criatividade. Vozes.

Minayo, M. C. S. (2017). Sampling and saturation in qualitative research: consensuses and controversies, Revista Pesquisa Quatitativa, 19(2), 1-12. https://editora.sepq.org.br/index.php/rpq/article/view/82/59 Portuguese

Ministério da Educação (2015). Instituições de educação superior e cursos cadastrados. Ministério da Educação. http://emec.mec.gov.br.

Ministério da Educação (2001). Resolução no 3, de 7 de novembro. Diretrizes Curriculares Nacionais do Curso de Graduação em Enfermagem.

Paro, C.A., Ventura, M. \& Silva, N. E. K. (2020). Paulo Freire e o inédito viável: esperança, utopia e transformação na saúde. Trabalho, Educação e Saúde, 18(1), e0022757. doi: 10.1590/1981-7746sol00227

Peres, C. R. F. B., Marin, M. J. S., Soriano, E. C. I. \& Ferreira, M. L. S. M. (2018). A dialectical view of curriculum changes in nursing training. Revista da Escola de Enfermagem da USP, 52, e0339. doi: 10.1590/s1980-220x2017038003397

Soriano, E. C. I., Peres, C. R. F. B., Marin, M. J. S. \& Tonhom, S. F. R. (2015). State nursing courses in São Paulo forward the curriculum guidelines. Revista Mineira de Enfermagem, 19(4), 965-979. doi: $10.5935 / 1415-2762.20150074$

Tonhom, S. F. R., Costa M. C. G., Hamamoto, C. G., Francisco, A. M., Moreira, H. M.\& Gomes R. (2014). Competency-based training in nursing: limits and possibilities. Revista da Escola de Enfermagem da USP, 48(esp 2), 213-220. doi.org/10.1590/S0080-623420140000800031

Ximenes Neto, F. R. G, Lopes Neto, D., Cunha, I. C. K. O., Ribeiro, M. A., Freire, N. P., Kalinowski, C. E., Oliveira, E. N. \& Albuquerque, E. M. N. (2020). Reflections on Brazilian Nursing Education from the regulation of the Unified Health System. Ciência \& Saúde Coletiva, 25(1), 37-46. doi.org/10.1590/1413-81232020251.27702019 